\title{
WETLANDS OF DHAKA CITY: ITS PAST AND PRESENT SCENARIO
}

\author{
Md. Shahidul Islam ${ }^{1}$, A. K. M Shahabuddin², M. Mostafa Kamal ${ }^{3}$ and Raquib Ahmed ${ }^{4}$ \\ ${ }^{1}$ Assistant Professor, Department of Geography and Environment, Pabna College, Pabna- 6600, Bangladesh \\ ${ }^{2}$ Deputy Secretary, Ministry of ICT, BCC Bhaban, Agargoan, Dhaka, Bangladesh. \\ ${ }^{3}$ Associate Professor, Institute of Bangladesh Studies, University of Rajshahi, Rajshahi 6205. \\ ${ }^{4}$ Professor, Department of Geography and Environmental Studies, University of Rajshahi, Rajshahi 6205.
}

*Corresponding author; email: shahidul_rubd@yahoo.com

\begin{abstract}
Landscape of Dhaka city, one of the fastest growing mega cities in the world, is undergoing continuous changes and modifications due to unplanned hasty urbanization process. As the growth of urbanization taking place at an exceptionally rapid rate the city is unable to cope with changing situation due to internal resource constraints and management limitations. Dhaka city endowed with a large number of water-bodies both big and small, which includes river, khals, lakes, flood plain low-lying areas etc. However, pre and post-urban changes of water-bodies in the city were studied using topographic map of 1960 and a satellite image of 2008 (ALOS VNIR 2008) through GIS and remote sensing technique. It is found that in 1960 total areas of water-bodies and lowland were 2952.02 and 13527.58 hectares. But in 2008 total areas of waterbodies and lowland found 1990.71 and 6414.57 hectares. Categories (water-bodies and lowlands) of wetland areas were decreased harshly. Study shows that water-bodies and lowland areas were decreased $32.57 \%$ and $52.58 \%$ that means more than $49 \%$ of the wetland areas decreased over the period 1960 to 2008. The changes of low-lying areas in the south-western corner extending towards Mirpur, Muhammadpur and PallabiCantonment areas and filled for the urban development. The part of Gulshan and Dhanmondi lakes has also been reduced; and some of the channels of Motijheel areas are not identifiable at present. The growth of urban infrastructures have been taking place in unplanned way; as a result it destroyed natural drainage systems, fill-up the water-bodies, causing water-loggings during rainy season in various part of the city. This study revealed that immediate necessary steps should take by concern authority to prevent from various disasters that might be occurred due to unwise and unplanned wetland changes.
\end{abstract}

Key words: Wetland, wetland change, waterlogging, Dhaka city, GIS,

সারাংশ: পৃথিবীর দ্রংত বর্ধনশীল মহানগরগুলির মধ্যে অন্যতম ঢাকা শহরের ভূচিত্র অপরিকল্পিত ও দ্রংত বর্ধনশীল নগরায়নের ফনে প্রতিনিয়ত পরিবর্তন রুপান্তর হচ্ছে। অতি দ্রংত নগরায়ন প্রক্রিয়ার ফলে ঢাকা শহর তার অভ্যন্তরীণ সম্পদের অভাব ও ব্যবস্থাপনার সীমাবদ্ধতার কারণে ভূচিত্র পরিবর্তনের অবস্থাকে নিয়ন্ত্রণ করতে পারছে না। ঢাকা শহরে ছোট-বড় অসংখ্য জলাশয় ছিল, যেমন নদী, খাল, হুদ, নিম্নভূমি ইত্যাদি। এই সকল জলাশয় ঢাকা শহরের নিষ্কাশন ব্যবস্থায় যেমন গুরুত্পূপূর ভূমিকা রাখতো তেমনি প্রাকৃতিক পরিবেশের জন্যও ছিল খুবই গুরুত্বপূর্ণ। কিন্ত দিন দিন ঢাকা শহরের জলাশয়গুলোর পারিসরিক বিস্তার সংকুচিত হয়ে আসছে। এই গবেষণার উদ্দেশ্য হলো ঢাকা শহরের জলাশয়গুলোর বর্তমান পারিসরিক বিস্তার, পরিবর্তনের ধরন ও প্রকৃতি সনাক্তকরণ এবং পরিবর্তনের ফনে সৃষ্ট প্রভাব নিরূপণ। গবেষণা কাজে ব্যবহৃত তথ্য সগ্রহের জন্য ভূসংস্থানিক মানচিত্র, উপগ্রহ চিত্র ব্যবহার করা হয়েছে। গবেষণায় ব্যবহৃত বিভিন্ন মানচিত্র ও স্যাটেলাইট ইমেজ বিশ্লেষণে দেখা যায় যে, ঢাকা শহরের জলাশয়গুলো খুব দ্রংত পরিবর্তিত হচেছ, ১৯৬০ সালে জলাশয় ও নিম্নভূমির পরিমাণ ছিল যথাক্রমে ২৯৫২.০২ ও ১৩৫২৭.৫৮- হেক্টর, কিন্ত ২০০৮- সালে সেই জলাশয়ের পরিমাণ এসে দাঁড়ায় ১৯৯০.৭১ হেক্টর এবং নিম্নভূমির পরিমাণ দাঁড়ায় ৬৪১৪.৫৮- হেঁ্টর। সুতরাং ১৯৬০-২০০৮- সাল পর্যন্ত মোট জলাশয়ের পরিমাণ হ্রাস পায় ৩২.৫৭\% এবং নিয়ভূমির পরিমাণ হ্হাস পায় ৫২.৫৮\%। জলাশয় পরিবর্তনের ক্রমবর্ধমান এই ধারা ঢাকা শহরের পানি নিষ্कাশন ব্যবস্থার মারাত্বক পরিবর্তন ঘটাচ্ছে যার ফনে জলাবদ্ধতা সমস্যার মত পরিবেশগত বিভিন্ন প্রভাব পড়ছে। যদি জলাশয় পরিবর্তন বন্ধের জন্য সরকার এখনই দ্রংত প্রয়োজনীয় ব্যবস্থা গ্রহণ না করে তবে অদূর ভবিষতে ঢাকা শহর একটি প্রাকৃতিক দূর্যোগের শহরে পরিণত হতে পারে।

\section{Introduction}

Dhaka city, the capital of Bangladesh, is situated in the central part of the country. It has one of the fastest urban growth rates among the developing countries (UN 1999; Alam and Rabbani 2007) and home to more than 10 million people (BBS 2004). The landform of the city is characterized by the Madhupur Tract an elevated Pleistocene terrace (Morgan and McIntire 1959) that stands higher than the neighboring floodplain and low-lying marshlands. The land cover of the city is being modified extensively by the progressive urbanization (UN 1999). The rapid rise in urban population is a major drive to the development of infrastructure and services, including road-network, water supply, sanitation, sewerage and drainage services and hence expansion of the city towards the surrounding floodplain and low-lying areas.
The relief controlled landforms of the area were efficiently drained via streams and canals (local term 'khals') (JICA 1991) to the floodplain and low-lying area and ultimately to the downstream via large rivers. These canals, wetlands and depressions have been filled up by new urbanization, both in and around the built-up city area (JICA 1991; Kamal and Midorikawa 2004). These unplanned urbanizations have been destroying the water-bodies and flow-paths causing rainfallflooding and drainage congestion in many locations in the city (Chowdhury et al. 1998; Reza and Alam 2002). Filling activities, embankments and roads are being compartmentalized the wetlands and water-bodies and hence obstructed the natural drainage. There is a general observation that the wetlands and other waterbodies in Dhaka city is greatly reduced over the decades due to progressive urbanization (Kamal and Midorikawa 2004; Dewan and Nishigaki 2006). There 
were number of studies mentioned about the land filling activities and their effects on the drainage congestion and water-logging (Kamal and Midorikawa 2004; Shams 1999; Chowdhury et al. 2001; and Tawhid 2004). The city at the moment, particularly, during the heavy downfall in the wet-season gets water-logged (Tawhid 2004). Thus almost one third of the city including Motijheel, Shantinagar, Rajarbagh, Mouchak, Najimuddin road, Jigatola, a few parts of Dhanmondi, Kazipara, Paikpara, Bijoy Sarani and others go under water (Kamol 2009). A number of canal/ khals, narrow creeks interweaved allover since long. In the historical past of the city, Dhaka was regarded as the Venice of east or the city of channels (Dani 1962). The situation has turned very bad in recent years. Main streets now go under a meter of water after heavy monsoon shower (Islam 2005). The scenario would be worsening with time as erratic and intense rainfall events postulated (Islam 2009) to be increasing their frequencies due to climate change (Alam and Rabbani 2007).

Rivers around the city gets the storms-drainage and other low grade (mostly households) liquid waste, and also liquid waste from the industries. These liquids and other pollutants have been polluting the river water (Rahman and Hossain 2008). Wetlands of the city could play a significant role in reducing the pollutants loads if the wetlands were designed as retention basin (Wang 2006 and Whigham 1999) for the urban drainage to the rivers. Plants and soils in wetlands play a significant role in purifying water, removing high levels of nitrogen and phosphorus, and in some cases, removing toxic chemicals through biogeochemical cycling and storage (Wang 2006).

However, there are very few studies (Chowdhury et al. 1998; Reza and Alam 2002) have been done on the changes of the wetlands in the city. Chowdhury et al. (1998) pointed on the elimination of local water storage and consequences in the mid-central western part of Dhaka city. Reza and Alam (2002) showed the changes of wetlands in the western half of the city. Both the studies concentrated on the western part of the city while none look over the whole city specifically, to the eastern part. History of urban growth (Tawhid 2004; Islam 1996) in the region demonstrates that the city is being expanding to the eastern part, low-lying areas, in the recent decades. These urban expansions are mostly occurring on to the wetlands in the eastern part, which were suggested to act as retention basin for urban drained water (JICA 1991). It is evident from the literature that the floodplains and low-lying areas were not under any sort of urban infrastructure during 1960s and urban growth in the area boosted during the 1990s. The objective of the present work is to map and distinguish the differences of the wetlands between the pre- and post-urbanization in Dhaka city. In this context a topographic map of the year 1960 (as preurbanization) and satellite imagery of 2008 (as post urbanization) were used to map and quantify the disparity between wetlands (lowland and water-bodies) applying remote sensing and GIS as a tool. A topographic map for the year 1960 was chosen because of the unavailability (at least with the current source) of a satellite image for that time.

On the other hand, there is no specific map or welldocumented records of wetland in Dhaka city. The land grabber takes this opportunity to encroach the wetlands. It is an urgent need to identify the wetlands of Dhaka city and evaluate its present Scenario.

\section{Objectives of the Study}

The specific objectives of the study can be set as follows:

- To identify the wetlands of Dhaka city and evaluate its present scenario;

\section{Location of the Study Area}

Dhaka city is located in the central part of Bangladesh and situated on the northern bank of Buriganga; the Balu and the Shitalakhya rivers in the east; Tongi Khal in the north and the Turag river in the west. The study area lies between $90.35^{\circ}$ and $90.50^{\circ}$ east longitudes and $23.70^{\circ}$ and $23.90^{\circ}$ latitude and an aerial extent of 297 $\mathrm{km} 2$ (Figure 1). These rivers are connected to the Ganges-Brahmaputra River system (locally known as the Padma-Meghna-Jamuna River system and also include the Old Brahmaputra river) flowing towards southeast from the all sides of the bigger neighboring region of the study area. The bigger area is closely dissected by number of rivers and khals which are hydro-logically connected to these major rivers. The area represents mostly flat land with slight undulations and stands few meters higher than the surrounding area. A large part of this city is covered by low-lying depressions. 


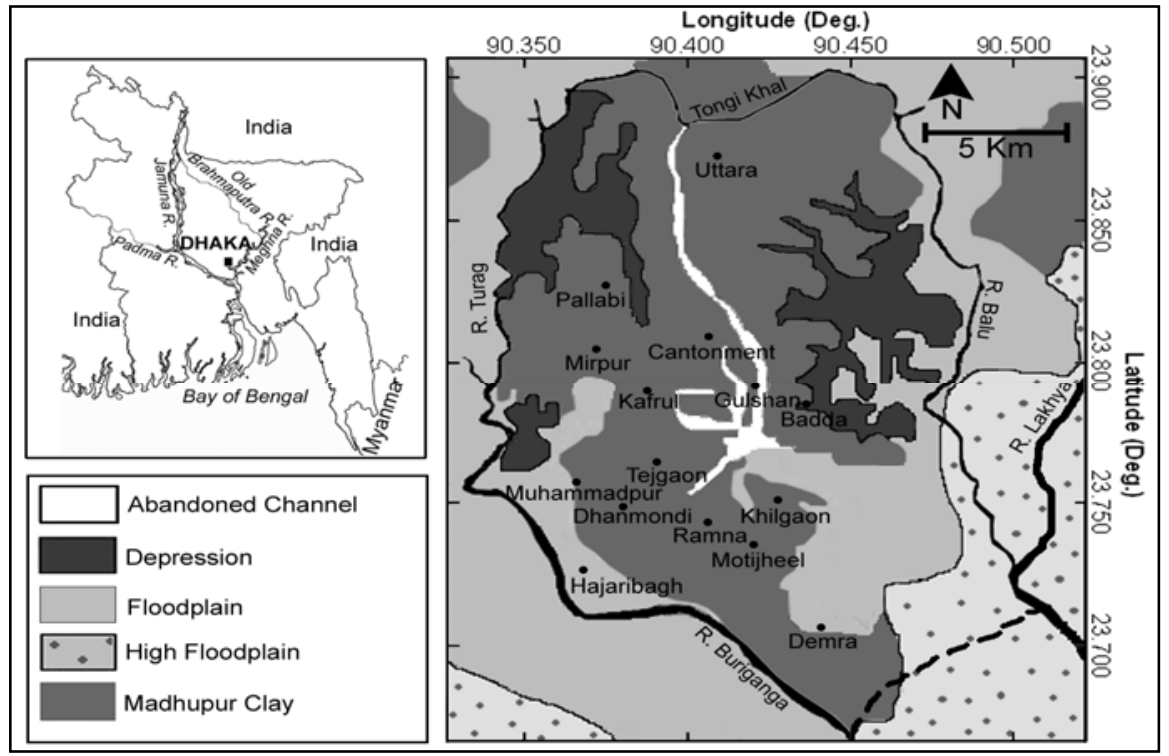

Figure 1 Location ot the study area along with prominent Iandtorm types Source: Adopted trom Alam et al. (1990)

The metropolitan and its surrounding areas are covered with Pleistocene Madhupur clay and Holocene sediments of floodplain origin (Alam et al. 1990). Holocene sediments are forming different types of landform in the area like: abandoned channels, depression, floodplain and high floodplain (Figure 1). The western part of the investigated area is the main old city founded on the semi-consolidated Pleistocene sediments (Madhupur clay), slightly elevated than the extreme western boundary, which is covered by the Buriganga-Turag floodplain sediments of Holocene to recent time (WASA 1991). The eastern boundary is mainly covered by the floodplains of the Balu River. These floodplains are characterized by low-lying depressions and marshy areas which remain inundated during significant period of the year. To the east, the low-lying area (depression and floodplain in Figure 1) is covered with recent flood-plain deposits. There are number of lakes, channels and khals in the city, which are connected to the surrounding rivers. The city is crossed by several faults and lineaments which are possibly controlling the orientation of rivers and streams (Khandoker 1987). The downthrown subsiding blocks are hosting marshy and swampy lands i.e. wetlands habitats (UN 1999; WASA 1991).

The storm runoff accumulates in the low-lying areas, flows through khals and local rivers and ultimately discharges to the major rivers. These lowlands and wetlands are performing important drainage function by storing storm water and keep the relatively higher lands free from rainfall flooding (Chowdhury et al. 1998).
The area has a tropical monsoon (May to October) climate like other part of the country with an average precipitation of $2000 \mathrm{~mm} /$ year.

\section{Material and Methods}

\section{Defining Wetland}

The RAMSER convention has defined wetlands as "areas of marsh, fen, peat land or water, whether natural or artificial, permanent or temporary, with water that is static or flowing, fresh, brackish or salt, including areas of marine water the depth of which at low tide does not exceed six meters" (Dugan et al. 1990). According to Khan et al. (1994) wetland holds water for a significant duration sufficient to support organism adapted to life in inundated or saturated soil condition and consists of wide variety of types ranging from lakes, rivers and coastal forest to deepwater paddy fields and ponds. The built-up area of the city is traversed and surrounded by wetlands of different types. For the purpose of the study, these wetlands are subdivided into three categories:

1) Lowlands (means open water body) comprises marshy and peaty in undated (during significant part of the year) low-lying areas of the TuragBuriganga and the Balu floodplains.

2) Permanent water-body includes the ponds, lakes and connecting canals of different water-bodies in the city area.

3) Fluvial water-body denotes the surrounding rivers (Turag, Buriganga, Balu River and Tongi Khal) of the city. 
It is however, analyses were done for the lowland and permanent water-bodies only. Third category was not included in the analysis as because this needs to be done with high resolution imagery with extensive field verifications. This is also out of the scope of the current work.

\section{Data and Software Used}

In order to compare the water- bodies, topographic map of 1960 and ALOS VNIR-Visible satellite image of 2008 were used. Topographic map (1:50000) of 1960, has been considered for pre-urban state water- body analysis. On the contrary, a multispectral ALOS VNIRVisible satellite image acquired in 21 November 2008 with 10 meters spectral resolution has been used to map the water-bodies of urbanized state of the city. The exact timing of the topographic map has not been found with the image. However, it was revealed from the source organization that these maps taken during the dry season around November-December time of the year. The temporal variation in the extent of the waterbodies is assumed to be insignificant over 1-2 months, at least for city-wide change analysis. However, this could be a source of uncertainties in the current analysis, in addition to the comparison of the two different spatial resolutions of the images. The data layers (containing the areas of water- bodies) of two years were overlaid to reveal the changes.

There are two strips of the topographic map to covers the study area. The topographic map did not have cartographic standard and three dimensional geographic distortions were found. The study has been carried out under the framework of Geographic Information System (GIS). The Image Processing tasks have been carried out using Earth Resource Data Analysis System (ERDAS) 8.4 image processing software (Leica Geosystems Geospatial Imaging, LLC, Norcross, USA). Data on wetland features has been extracted using ERDAS imagine software. GIS analysis has been carried out using ArcInfo software (ESRI, Redlands, California, USA) and the outputs have been generated using ArcGIS 9.3 software (ESRI, Redlands, California, USA).

\section{Data Processing and Analysis}

Data processing and analysis for the study is illustrate using a flow chart as in Figure 2. The ALOS VNIR-Visible satellite image of 2008 was collected in ERDAS Imagine (*.img) format. This image was loaded into computer memory directly from the CD (compact disk). Topographic map of 1960 were collected in the bitmap (*.bmp) format. These photographs were imported to (*.img) format to process the data using ERDAS Imagine software.

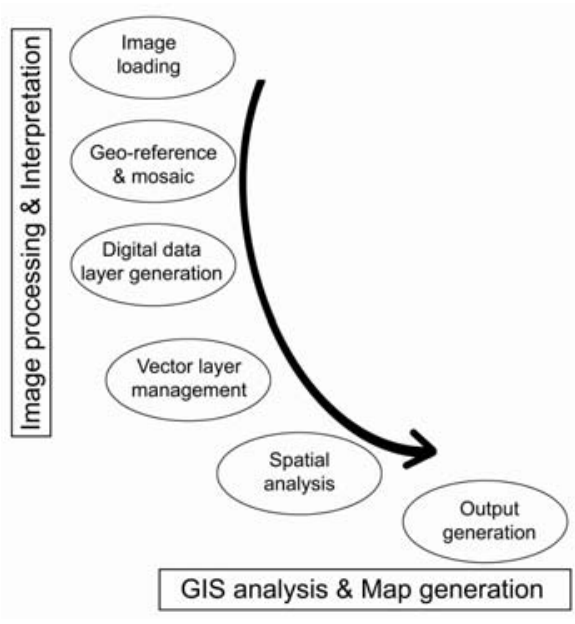

Figure 2 Data processing and analysis stages followed during the study

Geo-referencing was performed to render real world coordinates to the images to avoid geometric distortion. Topographic map of 1960 contain multi-scale geometric distortion. The satellite image of 2008 does not contain multi-scale geometric distortion; thus second order polynomial transformation was used for this image. For calculating the transformation matrix, 16 Ground Control Points (GCP) were used. The GCPs were derived from a geo-referenced image. Remarkable features, like road junctions, river bend etc., common to both images (1960 and 2008) were used as GCPs. In case of rivers or lakes, GCPs were chosen in the middle of the channel. Because most of the (spatial) changes to these features occurred at the boundaries of the channels or lakes. It should point-out here that the lakes in the study area are also channel-like (relict channel) features. The projection system used for the images was Lambert Conformal Conic (LCC). The geo-referenced two strips of topographic maps were mosaic to obtain the image of the whole study area. Then thematic data layers were generated from the geo-referenced images using on screen digitization technique. Data layers were generated in ArcInfo vector format using the digitization tools of ERDAS Imagine software. ArcInfo vector data layers generated from the images to render the GIS standard of the data. The spatial analyses of the thematic data layers were performed to generate composite data layers from the two images. Topographic maps coverage of individual type of water- body for the year 1960 and 2008 was calculated and the disparities between them were estimated. Changes were illustrated through map generation using ArcGIS soft-ware as described in result section. 


\section{Results Lowlands}

Analysis of topographic map of 1960 revealed (Figure 3) that most of the eastern part of Dhaka city is covered by lowlands in the form of the marsh-land or peaty areas of the Balu River floodplain. Western boundary of the city is also covered by the marshy lowlands. These lowlands in the west of Muhammadpur and northwest of Pallabi are generated within the floodplain of the Buriganga and the Turag river. Marshy land of the Turag floodplain area has extended into the built-up areas towards the northwest part of the city near Pallabi. The total lowland area is measured as 13527.58 hectares (Table 1). Satellite image of 2008 (Figures 4 and 5) shown that lowlands coverage areas of eastern part of the city reduced and become sporadic. The lowlands in the western boundary of the city are also reduced and become irregular. The total lowland area is measured 6414.57 hectares in 2008 (Table 1).

Table 1 Summary of analysis on the changes in wetland in the year 1960 and 2008

\begin{tabular}{l|c|c|c|c|}
\hline Categories of wetlands & 1960 area (ha) & 2008 area (ha) & $1960-2008$ area changed (ha) & \% of changed Area \\
\hline Permanent water-bodies & 2952.02 & 1990.71 & -961.31 & 32.57 \\
Lowlands(open water-bodies) & 13527.58 & 6414.57 & -7113.01 & 52.58 \\
Total & 16479.87 & 8405.28 & -8074.59 & 49.00 \\
\hline
\end{tabular}

Source: Analysis from the Figure 3 and 4

Note: (+) or (-) sign indicates direction of change

\section{Permanent Water Body}

Lakes, channels and khals, which are visible on the images, have been identified. The permanent waterbodies on the topographic map of 1960 are more prominent than 2008. Analysis and observation for permanent water-bodies on 1960 image show that the Gulshan Lake, Dhanmondi Lake and Ramna Lake are highly visible (Figure 3). Some channels and/or khals are also identified in different parts of the city. Channels are located in the northeastern, eastern, southwestern, southern and north-western corner of the city. The total areas of permanent water-body are measured 2952.02 hectares (Table1). Analysis of satellite image of 2008 (Figure 4 and 6) for permanent water body shows that the areas of lakes (Gulshan and Dhanmondi) have shrunken and narrowed down. Some khals and channels are not identifiable or missing in the southwestern (Muhammadpur) and southern (Motijheel) area of the city and the total area permanent water-bodies is measured 1990.71 hectares in 2008 (Table 2).

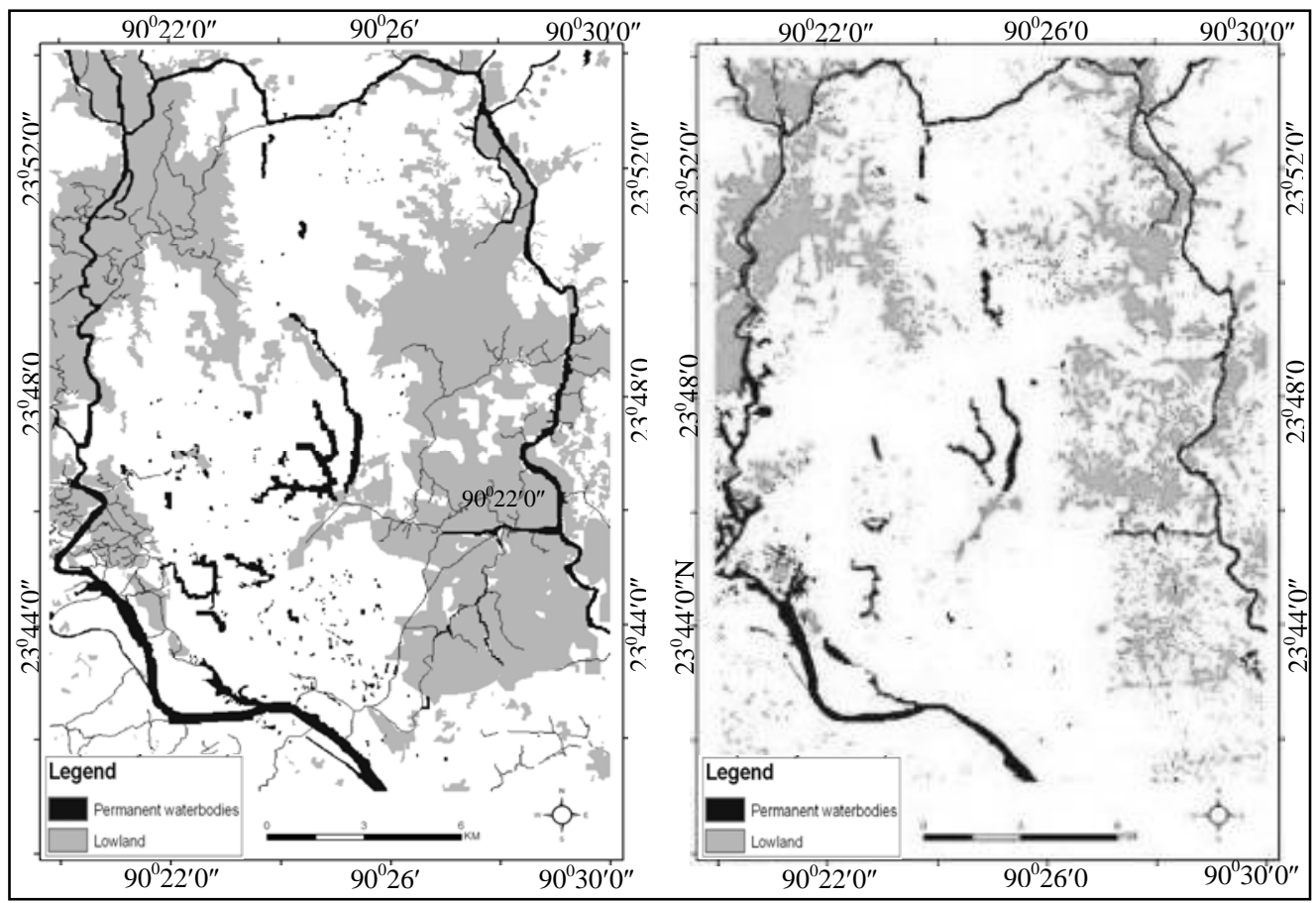

Figure 3 Different types of wetlands in the year 1960 Figure 4 Different types of wetlands in the year 2008 

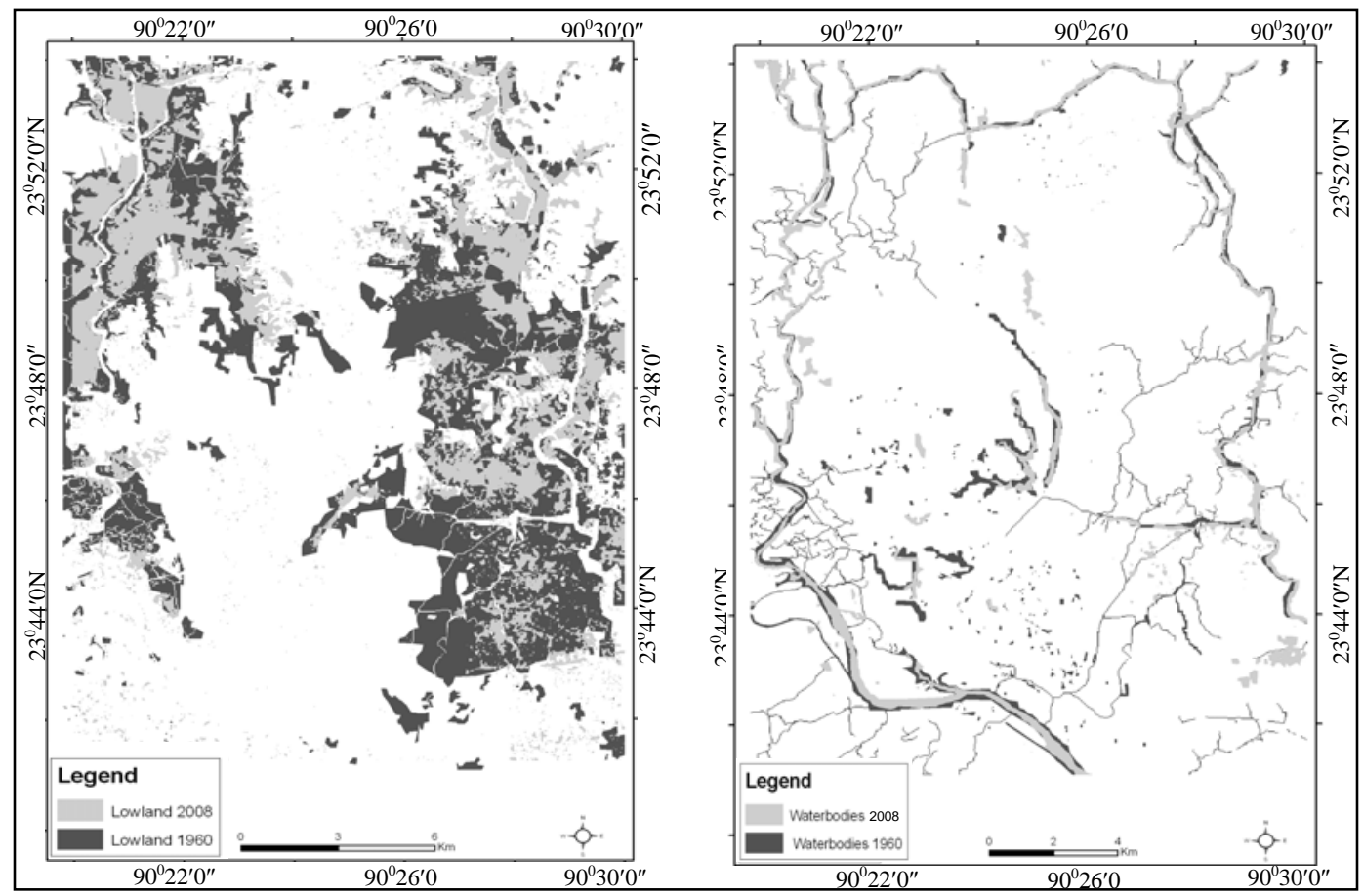

Figure 5 Overlay of lowlands of the year 1960 and 2008, Figure 6 Overlay of water-bodies of the year 1960 and 2008 , showing the differences. showing the differences.

Source: Prepared based on topographic map 1960 and ALOS, VNIR-Visible satellite image, 2008

\section{Discussion}

\section{Reduction of Wetland Area}

Analysis revealed that area covered by wetlands in the city significantly reduced over the period 1960 to 2008 (Table 2). In 1960, the area of the lowland was 13527.58 hectares, which became 6414.57 hectares in the year 2008. The amount of the lowlands reduction is 7113.01 hectares. The wetlands in the south-western corner retreat towards the Turag river in between Mirpur and Muhammadpur area. Minor reduction of the wetland has been occurred in the Pallabi-Cantonment area as well (Figure 5), where low-lying areas were filled and leveled for the urban extension. The reduction of wetland in this area closely matches the ground filling in Dhaka city as described in earlier works, e. g. (Kamal and Midorikawa 2004; and Shams 1999).

The area of the permanent water-body was found to be 1990.71 hectares in the year 2008 which was 2952.02 hectares in 1960. The reduction of area in the permanent water- body was 961.31 hectares (Table 2). The shape of the water- bodies remains almost same; but the widths of the lakes - Gulshan and Dhanmondi have been reduced (Figure 6). Permanent water- body has been mostly changed by the land filling and storm and waste water laid deposits. There are high-rise buildings constructed by the bank of these lakes encroached part of the lake area in some parts. Some of the channels in southeastern neighboring region of Motijheel area are not identifiable or missing in the 2008. These are narrowed or lost due to either encroachment or acquisition by government for construction of roads, box culverts or underground drains. These require ground truth and/or onsite mapping.

Urban expansion has been encroaching in the low-lying areas to cope with population growth, is the main reason for the reduction of wetland areas in the city. From the history of growth of the city, e.g. (Islam 1996) demonstrated that the city was remaining almost same up to the late 1980s. Earlier study, Reza and Alam (2002) showed that area of water-bodies in the western part of the city reduced to $91 \%$ of 1963 in 1990, which dropped to $63 \%$ of 1963 in 2000. In the present study the change in lowland areas (open water- bodies) over the entire metropolitan area is calculated to be reduced by 53\% in the year 2008 compare to 1960. It can be opined that this reduction happened in the 1990s too. From the earlier work e.g. (Dewan and Nishigaki 2006) it is vivid that the city areas in the early 1990s were extending the western low-lying areas while in the late 1990s and as well as in the 2000s the urbanization activities redirected to the eastern wetlands. 


\section{Significance of Wetland}

Wetlands associated with river floodplain system capture and retain water, reducing the duration and severity of floods (Dugan et al. 1990). Permanent wetlands intercept surface flow and slow it down, reducing the potential for floods. In the city development master plan (JICA 1991), wetlands in the eastern part were proposed as retention basin, which has long been acting so to keep Dhaka free from rainfall storm flooding. The unplanned urban extension into these wetlands may be causing water-logging problem during wet-season in the eastern part of the city (Dewan and Nishigaki 2006). This unplanned urbanization has also resulted into compartmentalization of the wetland. Due to poor connectivity between the different compartments of the wetland are causing water-logging in the built-up area as well. Water quality of the wetland would have been progressively deteriorating as wetland gets patchy and isolated.

The major lakes are Dhanmondi lake, Ramna lake, Gulshan lake and Cresent lake. In the early sixties Dhanmondi residential area was developed around the Dhanmondi lake which was a natural drainage line. To enhance the beauty of the area it was dug out and deepened in an area of 550 acres of land and trees were planted around the lake (Sultana 2005). A walkway has built adjoining the bank of the lake which protects the bank of the lake from encroachment.

The extent of groundwater recharge by a wetland is dependent upon soil, vegetation, site, perimeter to volume ratio, and water table gradient (Winter 1999). The soil under most wetlands in Dhaka city is relatively impermeable; however, perennial presence of these wetlands may signify their potential contribution to groundwater recharge. Moreover, some wetlands particularly around the built-up area have lesser thickness of silt clay at the bottom; have the higher potential to groundwater recharge. The wetlands around the built-up area and rivers around the city have found to play a significant role in the groundwater recharge (Ahmed and Burgess 2003). Demand driven abstraction of groundwater in the city has been failing to balance with amount of recharge in the recent years leading declining groundwater level and aquifer dewatering in many parts (Hoque et al. 2007). This may have been further enhanced by the reduction of wetland area.

\section{Conclusions}

Infilling of natural channels and lowlands for urban infrastructure have been reducing the area, and compartmentalizing the water-bodies which causing water-loggings and flood hazards in various parts of the city. Among the different types of water-bodies marshy and peaty inundated low-lying areas, natural retention basin around the city, are the hardest hit by the unplanned urbanization. In 1960, the total area of marshy and peaty inundated low-lying areas was 13527.58 hectares, which was found to be 6414.57 hectares in the year 2008. Over the last 48 years (1960 to 2008) $53 \%$ of the wetlands have been reduced. If the reduction rate (148 hectare/year) remained in place, the total wetland area in the year 2020 would be 4638.57 hectares. This means the wetland area may have reduced further from ' $53 \%$ of 1960 ' in 2008 to ' $34 \%$ of 1960' in 2020.

Most of the wetland losses in the eastern part of the city are associated with an urgent need for shelter by the less favored urban population, and growth takes the form of informal settlements. In the long run these informal growths are destroying the wetland ecology, reducing the area, and segmenting the integrity of the wetlands. Wetland loss can be viewed in several ways. The most obvious type of wetland loss is the conversion of a jurisdictional wetland to a non-jurisdictional status (Whigham 1999). For an example, wetlands in the northwest corner of the city (between Kafrul and Mirpur on Figure 1) have been acquired for the development of government offices over the last two decades. These wetland losses could be stopped if these were jurisdiction wetland instead of non-jurisdiction type. In Dhaka city wetlands should be classified as jurisdictional, and conserved by a regulatory board to preserve and restore the wetlands.

It is true that urbanization in Dhaka city would not be stopped, but these should be based on further specific studies and understanding of the hydrological system of the area, not just demand driven unplanned expansion. In addition, drainage in Dhaka city is strictly controlled by land relief and hence by gravitational drainage. Special care should be given to the development and alteration of the existing water-bodies so that natural hydrological condition can cope to the artificial structural actions. Water management must be the first concern for any development in Dhaka city because of its natural settings, and almost all the time people suffer from water and/or for water in a growing mega city.

\section{References}

Ahmed KM and Burgess WG. 2003. "Surface water and groundwater interaction in Bangladesh hydrogeology," In Groundwater resources and development in Bangladesh - background to the arsenic crisis, agricultural potential and the environment, AA Rahman P Ravenscroft et al., Bangladesh Centre for Advanced Studies, University Press Ltd., Dhaka, Bangladesh. 
Alam MK, Hasan MS, Khan MR and Whitney JW. 1990. "Geological map of Bangladesh,” Geological Survey of Bangladesh, Dhaka, Bangladesh.

Alam M and Rabbani MG. 2007. "Vulnerabilities and responses to climate change for Dhaka," Environment and Urbanization. 19: 81-97.

BBS 2004. Statistical Yearbook of Bangladesh, Bangladesh Bureau of Statistics (BBS), Ministry of Planning, Government of People's Republic of Bangladesh, Dhaka.

Chowdhury JU, Rahman R, Bala SK and Islam MS. 1998. "Impact of 1998 flood on Dhaka city and performance of flood control works," Integrated Water Flow Model (IWFM), Bangladesh University of Engineering \& Technology (BUET), Dhaka.

Chowdhury JU, Kamal MM, Khan NI and Salam MA. 2001. "Impact of land use change upon storm water drainage and wetlands in the eastern part of Dhaka city," Integrated Water Flow Model (IWFM), Bangladesh University of Engineering \& Technology (BUET), Dhaka, Bangladesh.

Dani AH. 1962. Dacca: A Record of Its Changing Fortunes. Ahmed Hasan Dani, Dhaka.

Dewan AM and Nishigaki KTM. 2006. "Flood hazard delineation in greater Dhaka, Bangladesh using an Integrated GIS and remote sensing approach,” Geo-carto International. 21: 33-38.

Dugan PJ et al. 1990. "Wetland conservation: a review of current issues and required action," International Union for the Conservation of Nature and Natural Resources Gland (IUCN), Switzerland.

Hoque MA, Hoque MM and Ahmed KM. 2007. "Declining groundwater level and aquifer dewatering in Dhaka metropolitan area, Bangladesh: causes and quantification," Hydrogeology Journal. 15: 1523-1534.

Islam N. 1996. "Dhaka: from city to mega city,” The University of Dhaka, Dhaka, Bangladesh.

Islam N. 2005. Dhaka Now Contemporary Urban Development. Bangladesh Geographical Society (BGS), Dhaka.

Islam MN. 2009. "Rainfall and temperature scenario for Bangladesh," The Open Atmospheric Science Journal. 3: 93-103.

JICA 1991. "Master plan for greater Dhaka protection project (Study in Dhaka metropolitan area)," Japan International Cooperation Agency (JICA), Dhaka, 1991.

Kamal MM and Midorikawa S. 2004. "GIS-based geo-morphological mapping using remote sensing data and supplementary geoinformation: A case study of the Dhaka city area, Bangladesh,” International Journal of Applied Earth Observation and Geoinformation. 6: 111-125.

Kamol E. 2009. The Death of Canals. The Star, 22 May, 2009. A Weekly Publication of The Daily Star.
Khandoker RA. 1987. "Origin of elevated Barind-Madhupur areas, Bengal basin: Result of neotectonic activities,” Bangladesh Journal of Geology. 6: 1-9.

Khan MS, Huq E, Rahman AA, Huq S, Rashid SMA and Ahmed H. 1994. "Wetlands of Bangladesh," Bangladesh Centre for Advanced Studies (BCAS) in association with Nature Conservation Movement (NA-COM), Dhaka, Bangladesh.

Khan NI. 2001. Assessment of Water-logging Conditions Using Integrated GIS and Remote Sensing Techniques: A Study of Dhaka Mega City. Oriental Geographer, 45(2): 41-54.

Morgan JE and McIntire WC. 1959. "Quaternary geology of Bengal Basin, East Pakistan and India," Geological Society of America Bulletin. 70: 319-342.

Rahman S and Hossain F. 2008. "Spatial assessment of water quality in peripheral rivers of Dhaka city for optimal re-location of water intake point," Water Resources Management. 22: 377391.

Reza MG and Alam MS. 2002. "Wetland transformation in the western part of Dhaka city (1963-2000)," Bhugal Patrika (Journal of Geography). 21: 23-40.

Shams N. 1999. "Urban geology of Dhaka city for geo-hazard mitigation and development planning,” M. Sc. thesis, University of Dhaka, Dhaka, Bangladesh.

Sultana MS 2005. Analysis of Changing Scenario of Wetlands in Dhaka City Using Remote Sensing and GIS. PGD Project Report. Institute of Water and Flood Management (IWFM), BUET, Dhaka.

Tawhid KG. 2004. "Causes and effects of water logging in Dhaka city, Bangladesh,” TRITA-LWR Master thesis, Department of Land and Water Resource Engineering, Royal Institute of Technology (KTH). 75p.

UN 2007. "Urban geology of Dhaka, Bangladesh, economic and social commission for Asia and the pacific, Atlas of Urban Geology,” United Nation (UN), New York, 1999.

Wang L Wang WD, Gong ZG, Liu YL and Zhang JJ. 2006. "Integrated management of water and ecology in the urban area of Laoshan district, Qingdao, China," Ecological Engineering. 27: 79-83.

WASA 1991. "Dhaka regional groundwater and subsidence model," Dhaka Water Supply and Sewerage Authority (WASA).

Whigham DF. 1999. "Ecological issues related to wetland preservation, restoration, creation and assessment," The Science of the Total Environment. 240: 31-40.

Winter TC. 1999. "Relation of streams, lakes, and wetlands to groundwater flow systems,” Hydrogeology Journal. 7: 28-45.

Manuscript received on 19 September 2012 and revised on 28 November 2012 